\title{
ALEJANDRO LOSADA
}

\author{
POR \\ MARTIN LIENHARD - INEKE PHAF - JOSE MORALES SARAVIA. \\ Freie Universität Berlin
}

E1 19 de enero de 1985, en un accidente aéreo cerca de La Habana, muere Alejandro Losada ${ }^{1}$. Con él desaparece no sólo un destacado docente, investigador y teórico de la literatura latinoamericana, sino también, y quizás ante todo, un hombre de gran inquietud intelectual, capaz de concebir vastos proyectos de investigación, de suscitar y fortalecer vocaciones científicas entre sus alumnos y amigos, de potenciar al máximo el sentido crítico de sus interlocutores. Un hombre, sobre todo en los últimos años, poseído por una gran idea: la de hacer colaborar, en su proyecto de una historia social de la literatura latinoamericana, al mayor número posible de estudiosos preocupados, más allá del afinamiento de sus propias técnicas y de su prestigio personal, por el presente y el porvenir de los pueblos latinoamericanos. Siempre dinámico, a veces contradictorio, a menudo provocativo, Alejandro Losada supo demostrar, ante todo, una curiosidad insaciable y una paciencia infinita. La imagen que mejor evoca, para el que escribe (M. L.), su ser más entrañable, es la de su dedicación veraniega al oficio de albañil en su casa-molino de la trastierra ligur, Italia: sin fijarse lo más mínimo en el paso del tiempo ni en las dificultades de la empresa, Alejandro iba buscando por todos los alrededores las piedras más adecuadas y les iba dando poco a poco la forma exacta exigida por el lugar previsto de su colocación; así seguía, nivel tras nivel, hasta que la pared o lo que fuera alcanzara la forma, altura y dimensión que él, en su mente, ya le había asignado.

${ }^{2}$ Este trabajo reúne las contribuciones de dos investigadores formados por Losada, Ineke Phaf («Trayectoria vital») y José Morales Saravia («Planteamientos y desarrollos teóricos»), así como la de Martín Lienhard («Consideraciones finales»), quien se responsabiliza también por la coordinación. 
En las páginas que siguen, los autores quieren ofrecer una aproximación múltiple a la vida y la obra de quien fue, además de amigo, su maestro o colega.

TRayectoria Vital (I. P.)

E1 7 de mayo de 1936 nace Alejandro Losada en Córdoba, Argentina. A lo largo de sus años formativos se va gestando la motivación que lo 1levará a dedicarse exclusivamente al estudio de las literaturas latinoamericanas. De 1954 a 1958 cursa estudios de filología clásica en Córdoba y Santiago de Chile. En la Universidad del Salvador, Buenos Aires, se formará luego en filosofía (1959-1961) y en teología (1965-1967). Dicha Universidad, dirigida por los jesuitas, es en aquel entonces centro de un acendrado debate acerca del papel de los intelectuales en la sociedad argentina. Los primeros trabajos publicados versan sobre el Martín Fierro de José Hernández (Losada, 1967) y sobre algunos relatos de la guerra de frontera contra los indios en el siglo xIX (L., 1968). Su pasión por este doble núcleo temático -el gaucho-héroe y la constitución de la sociedad argentina en y por la violencia antipopular- nunca abandonará a Alejandro Losada. A su muerte, en efecto, él deja, entre otras cosas, una excepcional colección de documentos (periódicos, cartas, fotos, etc.) de y sobre José Hernández, que espera su clasificación y publicación.

Para exorcizar una para él traumática experiencia de asesoría gubernamental (1968-1970) en el período de Onganía, Losada escribe su único texto de ficción, la novela Andá cantále a Gardel (L., 1970), que figurará durante semanas en la lista de los best-sellers de las librerías bonaerenses. El narrador-protagonista de este relato, en buena parte autobiográfico, es un cordobés que va buscando en la capital la explicación de los males que sufre el país, males que él ve evocados en un tango de Carlos Gardel sobre la convulsiva entrada de Argentina al siglo xx:

\author{
Pero el siglo veinte \\ es un despliegue de maldad insolente \\ ya no hay quien lo niegue \\ Vivimos revolcaos en un merengue \\ y en un mismo lodo, todos manoseados...
}

Como funcionario del Ministerio de Educación y Cultura, el yo novelesco lucha contra una realidad detrás de la cual se perfila el pasado, el del Matadero de Echeverría con su matón Matasiete. Es en la actividad polí- 
tica y en su vida personal donde el doble del autor buscará una salida del conflicto a la vez individual y social que lo va devorando. Su pregunta final, lanzada al futuro, resume su desasosiego:

El complot está en todas partes y en todo momento. Hasta dentro mío. Igual que el matadero. Ahí viene. ¿Quién será, esta vez, el unitario?

Mucho más tarde, Losada dará una interpretación científica de ese difuso y opaco sentimiento de «complot»; el malestar se origina en la historia latinoamericana, «un proceso ininterrumpido que tiene como punto de partida el TRAUMA ORIGINAL que confiere especificidad a la sociedad y la cultura de la región y que llega hasta hoy» (L., 1985d).

En los círculos intelectuales de los años sesenta se va discutiendo el impacto, en el terreno cultural, de las relaciones de dependencia que sufre la «periferia» latinoamericana. Ante la ausencia de perspectivas positivas ofrecidas por la política del gobierno de turno, Losada optará definitivamente, abandonando sus veleidades políticas y artísticas, por la dedicación exclusiva a la investigación literaria.

En 1971 se traslada al Perú con quien será, desde entonces, su esposa: Ethel Salas. Como profesor asociado de la Universidad Nacional Mayor de San Marcos (Lima), empieza a desarrollar un trabajo científico sistemático. En su libro Creación y praxis (L., 1976a), fruto mayor de su actividad limeña, Losada concibe la literatura como una forma de praxis social y analiza, bajo este ángulo, a cinco narradores peruanos. Tomando como punto de referencia los presupuestos elaborados por G. Lukács, él los amplía en el sentido de la especificidad de los condicionamientos socioculturales en América Latina: actitud que le permite sustraerse a una imitación servil, neocolonial, de los teóricos europeos. Un artículo de 1975 define su posición científica: el investigador — dice- debe «tratar de fundar una praxis social, es decir, que ve, en su práctica científica, también un modo de instituir una relación concreta y activa con los hombres de su sociedad y una participación en sus conflictos» (L., 1975b). Al mismo tiempo que desarrolla su reflexión teórica, Losada materializa sus ideas en una praxis científica colectiva y abierta que caracterizará siempre, desde entonces, sus actividades. En Lima, todavía, él participa en la empresa - colectiva - de la puesta en marcha de la Revista de crítica literaria latinoamericana (1975).

En 1976 se le brinda la oportunidad de pasar, como profesor visitante, una temporada en el Institute of Latin American Studies de la Universidad de Austin, Texas. Su encuentro con el etnohistoriador R. Schaedel 
repercutirá profundamente en el giro «antropológico» que tomará su orientación científica.

A partir de fines de 1976 Losada se establece en Europa. En la Universidad de Erlangen/Nuremberg, donde se desempeñará como profesor visitante e investigador, pasará su doctorado (1978: L., 1983). En 1978 gana la cátedra de literatura en el Lateinamerika Institut (LAI) de la Universidad Libre de Berlín (occidental). Es en el ambiente interdisciplinario de esta institución donde concibe su gran proyecto de una historia social de las literaturas latinoamericanas. Desde entonces, su biografía coincidirá prácticamente con el progreso de esta empresa.

Los trabajos se llevan adelante en tres espacios institucionales distintos. En un coloquio interno, donde participan estudiantes, doctorandos y especialistas invitados, se discuten las estrategias de investigación, tomando como base la concepción losadiana de los «sistemas literarios» y el principio de un estudio comparativo de las literaturas en cinco regiones histórico-culturales: México, Caribe y Centroamérica, Brasil, Andes, Ríode la Plata. La incorporación de Brasil y el Caribe como regiones específicas de un conjunto subcontinental significa, para esa época, una innovación. Desde 1981, la investigación se centra en una comparación entre la región caribeña y la rioplatense, con vistas a definir la diferenciación sociocultural de las dos entidades y su articulación respectiva con la producción cultural europea. Dentro de este contexto se estudia especialmente el fenómeno paralelo de la «internacionalización» de las literaturas latinoamericanas y su tendencia a apropiarse las tradiciones populares, en general orales, heredadas, en parte, de la Europa medieval. En un trabajo reciente, Losada analiza en este sentido el Martín Fierro: el poema representa, a primera vista, una actualización de la épica medieval española; el personaje del héroe, con su concepción «catastrófica» de la historia, resulta, en cambio, un típico producto de la cultura popular latinoamericana, de su perspectiva informada por el impacto del «trauma original». Varias tesis realizadas por estudiantes y doctorandos del LAI atestiguan la efervescencia científica creada por la presencia de Losada en esa institución.

La dinámica de la colaboración con otros investigadores residentes o de paso en Europa desemboca, en 1982, en la creación de un segundo espacio institucional de investigación: la Asociación para el Estudio de las Literaturas y las Sociedades en América Latina (AELSAL). Como miembro fundador y presidente de esta asociación, Losada impulsa y orienta científicamente los congresos de Giessen, R.F.A. (1982) y de Travers, Suiza $(1983,1984)$, a los cuales acuden investigadores cada vez más numerosos de varios países europeos. A la muerte de Alejandro, la asociación queda bien consolidada. Una serie de publicaciones colectivas, impul- 
sadas por él, permiten apreciar el avance de la investigación (L., 1983e, 1985c, 1986).

Fuera del trabajo realizado colectivamente con sus alumnos del LAI y con los investigadores de AELSAL, Losada no descuida nunca un tercer espacio, el de los intercambios científicos a escala internacional. Ya en 1979 organiza en Berlín, conjuntamente con la profesora $G$. Beutler, un coloquio internacional sobre César Vallejo (L., 1981c). En tres congresos de la Latin American Studies Association (LASA) -Bloomington 1980, Washington 1982, México 1983 - organiza sendos workshops para presentar los últimos resultados y desarrollos metodológicos de su investigación. Todos estos esfuerzos tienden siempre a ampliar y a afianzar la presencia científica de su proyecto de una historia social de las literaturas latinoamericanas. La fuerza de irradiación personal de Alejandro acabará convirtiendo su casa en Berlín-Wannsee, su oficina en el LAI y su residencia rural en Liguria en verdaderos centros internacionales de debate literario entre docentes, investigadores, estudiantes.

En enero de 1985 -reconocimiento «oficial» de sus méritos en el campo de los estudios literarios latinoamericanos- Alejandro Losada es invitado a formar parte del jurado del premio Casa de las Américas, en La Habana. Lo alcanza la muerte cuando se dirige, desde ahí, a la capital de Nicaragua, donde iba a presentar un planteamiento renovado de los procesos literarios en el área caribeña (L., 1985d).

\section{Planteamientos y Desarrollos teóricos (J.M.S.)}

1. Los desarrollos y planteamientos que se expondrán a continuación tienen que ser situados dentro del marco de carencias y preocupaciones que afrontaba la disciplina latinoamericanista a finales de los años sesenta. Asumir polémicamente esta joven tradición de la disciplina para ponerla en duda o reformularla va a ser justamente uno de los ejes - y además un motor- de la actividad losadiana. Así, por ejemplo, sus trabajos sobre la narrativa peruana contemporánea (L., 1976a) o sobre el «romanticismo» en el Río de la Plata y el Perú (L., 1983a), que muestran muy claramente su preocupación por otorgar un status científico a estos estudios mediante la constitución de objetos de conocimiento pertinentes, estrategias de investigación operativas, metodologías procedentes, conceptos explicativos que pudieran dar cuenta de los fenómenos en toda su compleja evidencia.

2. Es en este sentido - lo que precede constituye a la vez el punto de partida y la perspectiva - como concebirá Losada la literatura y los fenómenos literarios dentro de la sociedad, no como un elemento separado 
al que se tendría que articular (y he aquí su polémica con ciertos desarrollos de la sociología de la literatura, o más lejos, con ciertas formas de la teoría del reflejo: L., 1976a), sino como un elemento, entre otros, que es también constitutivo de esa sociedad. Es en este lugar donde van a aparecer sus nociones de literatura como praxis social (es decir, como la actividad de ciertos grupos por la cual definen su vida y su pertenencia al mundo social), como institución social (aquello que crea y posibilita relaciones entre los hombres en el mundo social) y como dadora y creadora de un horizonte de existencia, es decir, como instauradora de sentido en ese mundo social.

3. También en este nivel de los que uno podría llamar presupuestos básicos, y en discusión con ciertos procedimientos de la disciplina «tradicional», habría que situar su insistencia en la noción de especificidad. Detrás de esta noción se encuentra el planteamiento de que las sociedades latinoamericanas, en su surgimiento, formación y constitución, demuestran una serie de características que les son peculiares; otro tanto ocurre con las diferentes praxis que las constituyen y, lo mismo, con la praxis particular llamada literatura. E1 lado polémico que encierra, por lo demás, esta noción apunta a la aplicación acrítica de los conceptos, las denominaciones, etc., surgidos en otros espacios culturales e históricos; por ejemplo, el uso de términos (incluido su significado) como romanticismo, vanguardia y otros (L., 1983a).

4. Menciónese aún que esta noción de especificidad trae consecuencias importantes en su aplicación a la propia región de estudio. Es ella la que permite establecer unidades espaciales menores con un carácter no sólo operativo. Es la delimitación de subregiones según los momentos de formación o constitución de las sociedades implicadas y también según sus productores y sus espacios culturales. En este sentido se tendría que hablar propiamente de literaturas latinoamericanas (arreglo de cuentas con las concepciones de la literatura latinoamericana como unidad o como parte de la «literatura universal»), pues las sociedades dentro de las que ellas han surgido estarían caracterizadas por su desarrollo desigual y por sus divergentes formaciones sociales. Así se hablaría de las subregiones del Cono Sur, Pacífico andino, Brasil, Caribe y Centroamérica, México, manejando estos términos con la debida elasticidad.

5. En cuanto a los planteamientos estratégicos, la propia trayectoria investigadora de Losada aclara algunos de ellos. Luego de un primer momento donde se podrían situar sus trabajos monográficos sobre el Martín Fierro, Ciro Alegría y José María Arguedas (L., 1967, 1974, 1975c), aparece la preocupación por extender el objeto de estudio tratando de saltar por encima de las limitaciones que mostraban las investigaciones de este 
tipo, que no lograban ofrecer sino una explicación parcial de desarrollos más amplios. Las nociones de conjuntos literarios y sistemas literarios son el resultado de este nuevo paso metodológico. El problema que afrontaba Losada era el de abordar estratégicamente la selección de textos y autores para tomarlos como objetos de estudio. Se trataba, pues, de echar mano del criterio de la representatividad, ya que operativamente parecía imposible abarcar todos los textos. ¿Qué había que entender por textos representativos? Los textos que constituían y representaban los momentos claves de cada proceso histórico-social. Pero estos textos, en una segunda operación, podían a su vez ser reagrupados sistemáticamente en subgrupos. Al cabo resultaban unos pocos sistemas que explicaban las diversas formas de conciencia de una formación dada, las diversas formas de organizarla o de implementar horizontes, así como las diversas funciones que cumplía cada uno de ellos (Losada, 1975a).

6. Menciónese aquí, de una vez, que la noción de modo de producción precedía a la de sistema literario. Losada la había aislado siguiendo una serie de pasos metodológicos. Se trataba, en primer lugar, de preguntarse por el sujeto productor de los textos. Aquí no se identificaba este sujeto con el autor, ya que por lo general denominaba la palabra «sujeto» un conjunto de autores o un «grupo» determinado. En segundo lugar, se procedía a aislar y describir el paradigma estético-cultural que este sujeto elaboraba, es decir, sus diferentes y distintos discursos. En tercer lugar, se preguntaba por las funciones que quería cumplir el sujeto productor con la elaboración de su paradigma estético-cultural. Solamente que estas funciones, en cuarto lugar, recibían su encuadre según en qué espacio social se encontrase el sujeto productor. Así, el espacio social de una sociedad tradicional resistente y de reestructuración exitosa, o el espacio social de una sociedad en crisis y en vísperas de una nueva etapa, o el de una sociedad diversificada y compleja de rasgos capitalistas, o el de una sociedad con una economía de enclave y sin lugar para instituciones culturales, debían ofrecer diversos sujetos productores, disímiles paradigmas estéticoculturales que cumplirían, por consiguiente, diferentes funciones. Pues bien, estas cuatro nociones constituirían el concepto de modo de producción cultural que se mencionó anteriormente (quede dicho que el orden de presentación no corresponde necesariamente a los pasos metodológicos de la investigación) (L., 1977).

7. Habiendo realizado estos procedimientos metodológicos y arribando a la noción de modo de producción, se trataba de reordenar esos conjuntos de textos en sistemas correspondientes. En este sentido, las investigaciones de casos habían conducido a aislar los siguientes: el sistema dependiente, por ejemplo, describía espacios sociales tradicionales rees- 
tructurados más o menos exitosamente, donde las instituciones culturales sólo existían en su supeditación a las capas hegemónicas y donde el sujeto productor tenía una existencia cooptada en su elaboración de un paradigma estético-cultural dirigido a dichos sectores. Este sería el caso de aquello que se ha dado en llamar el «romanticismo» peruano en la segunda mitad del siglo XIX. Otro ejemplo lo ofrece el sistema autónomo que describe sociedades tradicionales que no otorgan un espacio de desarrollo a los sujetos productores; éstos se ven en la necesidad de crearse un lugar propio y elaboran un paradigma donde justamente se impugna aquel orden que les niega su existencia. Sería el caso del «romanticismo» argentino o de la literatura llamada «de los proscritos». También tenemos el sistema marginal, con un sujeto productor moviéndose en el espacio social de una sociedad compleja y diversificada, desvinculado de los problemas de la hora y creador de un paradigma estético-social donde dominan los elementos de la desesperanza y la conciencia catastrófica. Es el caso de gran parte de las producciones literarias en el Río de la Plata de los años treinta; especialmente de lo que uno podría llamar el "grupo» Sur (Losada, 1984a). Además, el sistema social-revolucionario, desarrollado en espacios sociales que presentan, en una sociedad en crisis y en reestructuración, una apertura para un cambio; sus sujetos productores elaboran un horizonte alternativo al existente en una literatura cuya función es precisamente propugnar y producir este cambio. Se da en varias épocas, distintas en los diferentes países; por ejemplo, a partir de 1920 en cierta literatura producida en el Perú, especialmente la que surge alrededor del «grupo» Amauta. Otro sistema todavía sería el internacionalizado, sistema donde los productores ya no se encuentran en su espacio social de origen, sino que, sin dejar de elaborarlo, se han mudado a otros espacios sociales, metropolitanos, y han asumido esa nueva problemática y alcanzado nuevos públicos $\mathrm{y}$, por lo mismo, constituido nuevos paradigmas estético-culturales que cumplen nuevas funciones. Es el caso del movimiento de la negritud, de la literatura producida en las metrópolis en los años treinta por los grupos antifascistas, etc. (L., 1983b, 1984b).

8. A las preocupaciones que se encontraban detrás de estos desarrollos teóricos y metodológicos de Losada hay que añadir otra que se hace patente y se clarifica a principios de los años ochenta. Se trata de su proyecto de escribir, junto con un grupo de otros investigadores, una Historia Social de la Literatura Latinoamericana que llenara el vacío que a este respecto existía en la disciplina, dada la deficiencia de las Historias al uso. En este sentido se desarrollaron sus últimos trabajos tratando de resolver los problemas que una tal empresa supone desde el punto de vlsta teórico. Si la noción de sistema se manifestaba de suma utilidad para este proyecto, 
pues permitía trabajar con conjuntos de textos amplios, quedaba todavía por considerar el asunto de los cortes temporales, las sincronías y las diacronías, es decir, el problema de la periodización (L., 1981b, 1985a). Se trataba otra vez de superar las limitaciones de las periodizaciones que circulaban y que no hacían, en la mayor parte de los casos, sino trasladar sistemas existentes para otras regiones y aplicarlos al caso latinoamericano. En este sentido iban los planteamientos que enunciaban nociones como momentos o períodos. Momento se refería a los espacios temporales más o menos «largos», sean éstos de formación (momento formativo), de constitución (momento constitutivo) o de determinación (momento decisivo). Así, por ejemplo, el momento constitutivo podía ser, según la formación social enfocada, de reestructuración oligárquica, de liquidación de la herencia colonial, de articulación capitalista, etc. Por otra parte, la noción de período hacía referencia a unidades temporales más reducidas que se encontrarían dentro de los diferentes momentos. Así, para mencionar un solo caso, una vez que se instaura el momento de la liquidación colonial en el Río de la Plata, se podrían aislar los períodos oligárquicos ornamentales (segunda mitad del siglo XIX), de auge metropolitano (1900-1945) y de crisis del proyecto modernizador: los últimos veinticinco años (Losada, 1983a).

\section{CONSIDERACIONES FINALES (M. L.)}

En vez de arriesgarnos ahora a presentar una todavía prematura evaluación global del sistema conceptual elaborado por Alejandro Losada, preferimos esbozar aquí una serie de consideraciones tendientes a descubrir las motivaciones y finalidades que subyacen a este sistema, sus aportes a una ciencia general de los procesos culturales en América Latina y, también, algunas de las cuestiones que deja manifiestamente pendientes. Cabe subrayar, en primer lugar, su índole de sistema «abierto», capaz de transformarse constantemente bajo el impacto de los impulsos suscitados por el desarrollo de la reflexión en el campo de los estudios literarios y en las disciplinas vecinas: historia, antropología, sociología.

La historia, concebida no como una sucesión lineal y necesaria de épocas en el sentido positivista, sino como un proceso dialéctico, configura el trasfondo sobre el cual se mueve el pensamiento de Losada. Sus alusiones casi obsesivas a las rupturas históricas (opuestas implícitamente a la idea de un progreso continuo) y su manejo de un concepto de «tiempo largo», muestran una evidente convergencia, consciente o no, con las elaboraciones teóricas de la ciencia histórica contemporánea (Foucault, Braudel y 
otros). Como ya se vio, la especificidad del proceso histórico latinoamericano se cifra, para Losada, en la imagen del «trauma», que se refiere a la vez a la violenta ruptura de los «comienzos» (Conquista) y a la violencia estructural que siguen reproduciendo, desde entonces hasta hoy, los mecanismos de la relación de dependencia con respecto a los centros de decisión exteriores (colonialismo-neocolonialismo-imperialismo). La concepción histórica losadiana, basada ni en una simple cronología ni en la historia «política», sino en los procesos socioantropológicos, queda, pues, muy alejada de la que subyacía a la mayoría de las historias literarias latinoamericanas.

La presencia de un marco sociológico en los trabajos de Losada no constituye, en sí, ninguna innovación; inédita, en realidad, es su vinculación con el carácter específico de los procesos históricos latinoamericanos y con el conjunto, también específico, de los contextos que llamaremos «antropológicos».

Una reflexión de tipo antropológico constituye, sin duda, la base última del sistema losadiano; dicho de otro modo: su especulación teórica se inscribe en el marco, todavía latente, de una ciencia general de los procesos culturales latinoamericanos. El postulado básico de que las literaturas latinoamericanas no han de enfocarse como un apéndice atrasado de las europeas, sino como una expresión de un devenir histórico peculiar, deriva en definitiva del "relativismo cultural» defendido especialmente, sin aceptar la etiqueta, por Lévi-Strauss. En la medida en que Losada dedica una atención especial a las consecuencias culturales de las relaciones de dependencia (metrópolis/periferias), su elaboración evita, sin embargo, un aspecto ideológico del «relativismo cultural»: la tendencia a escamotear, precisamente, las relaciones coloniales o neocoloniales que diseñan, en el mundo moderno, el marco dentro del cual se desarrollan las diferentes culturas locales o «periféricas».

Otro postulado básico del sistema losadiano, el de la índole colectiva de toda producción y difusión cultural, se inscribe igualmente en el pensamiento antropológico moderno. En vez de considerar los textos como objetos de investigación autosuficientes, Losada subraya el carácter de práctica social que suponen las operaciones constitutivas - producción, recepción, transformación- del «fenómeno literario». El concepto de "grupo productor», llamado a sustituir al de «autor», vincula la concepción antropológica de una práctica colectiva con la definición sociológica del lugar de los intelectuales en las formaciones sociales modernas de América Latina.

La literatura, para Losada, no es, por otra parte, una práctica en sí, sino uno de las prácticas semióticas o «culturales» que realiza una socie- 
dad determinada; se entrevé, aquí también, una convergencia con un pensamiento antropológico que considera las sociedades como vastos y complejos sistemas de comunicación, cuya segmentación no tiene sino un valor metodológico y didáctico. La práctica literaria, inseparable de las demás prácticas comunicativas, constituye uno de los segmentos aislables para los fines de una investigación científica apoyada en un instrumental específico. Estos presupuestos implícitos explican el interés demostrado por Losada en distinguir, al lado de los sistemas expresivos cultos (como la "literatura ilustrada»), la existencia de sistemas estéticos populares basados en la oralidad, y en rastrear las relaciones de interdependencia entre los dos polos que se van desarrollando en el seno de las sociedades latinoamericanas. Un corolario de este enfoque es el rechazo, compartido hoy por numerosos investigadores, de una dedicación exclusiva a los géneros literarios canónicos (europeos) y la valoración de los discursos antaño considerados como no literarios (crónicas, testimonios, folletines, etc.).

Una orientación básicamente antropológica, todavía, subyace a la «regionalización» losadiana de los procesos histórico-culturales en América Latina. Su delimitación de las diferentes áreas, en buena parte coincidente con la que realizó, con otros objetivos, el antropólogo brasileño D. Ribeiro (As Américas e a civilização, 1970), toma en consideración, fundamentalmente, la historia «étnica» del subcontinente: áreas pobladas, dentro de los límites de los grandes estados prehispánicos, por indios y descendientes de indios (México, Andes); áreas caracterizadas por la importación masiva de esclavos africanos (Caribe, Brasil); área «desindigenizada» que sufrió, en épocas recientes, una multitudinaria inmigración europea (Cono Sur). Las respectivas constelaciones «étnicas», resultado y parte de los procesos históricos subregionales, imprimen, junto con las características de la configuración social, un sello particular a las prácticas culturales de cada área. La división del subcontinente en áreas históricamente pertinentes, articulada con una visión dialéctica de las relaciones de dependencia, es el hallazgo que permitió a Losada plantear en términos nuevos el problema de la periodización literaria: en vez de suponer la uniformidad, bajo la «influencia» de las corrientes europeas, de los procesos literarios latinoamericanos, Losada elabora, como lo señaló Morales en el capítulo anterior, una periodización asincrónica por áreas histórico-culturales.

El edificio teórico elaborado por Losada tiende a diseñar, como ya se apuntó, una antropología de las prácticas literarias en América Latina. Esta orientación epistemológica explica, sin duda, las conocidas reacciones negativas de los sociólogos literarios «ortodoxos», identificados con la concepción de la literatura (de los textos) como reflejo del devenir históricosocial, así como la de los filólogos — tradicionales o estructuralistas- que 
buscan en vano, en los trabajos de Losada, el esbozo de una poética o de una historia de las formas literarias en América Latina. El status de los textos concretos, en la teoría losadiana, no es, en efecto, el de objetos últimos de la investigación; basándose, por lo general, en los análisis ya realizados con las técnicas propias de la poética, ella considera los textos como signos de un sistema de comunicación más vasto que los trasciende y llega a constituir el verdadero centro de interés. El enfoque losadiano no niega, en realidad, la necesidad de construir una poética de los discursos literarios latinoamericanos, pero no asume la tarea de fundarla. La realización futura del gran proyecto losadiano de una historia social de las literaturas latinoamericanas exigirá, sin duda, una colaboración más estrecha entre los sociólogos o antropólogos de las prácticas literarias y los «poetólogos» latinoamericanistas.

Concluiremos esta evocación del perfil científico de la obra losadiana con algunas observaciones acerca de la que aparece como su motivación profunda. Losada no consideró nunca su actividad docente y teórica como el ejercicio autosuficiente, gratuito, de sus capacidades dialécticas. En su opinión, la investigación literaria se justificaba en la medida en que contribuía, aun mínimamente, a liberar la carga de «negatividad» -en el sentido sartreano- que poseían, según él, los productos más destacados de la cultura latinoamericana desde la época colonial. Las crónicas de la conquista, por ejemplo, afirmó en uno de sus últimos trabajos, formarían un único corpus con las obras más significativas de la literatura contemporánea (L., 1985a). Fabricar una historia de la literatura latinoamericana no equivalía, por lo tanto, a reinterpretar el pasado ni a diseñar la arquitectura de un museo de las prácticas literarias desusadas, sino que suponía un gran esfuerzo para rescatar, en todas las épocas, las prácticas y los significados que, una vez integrados a nuestro horizonte contemporáneo, podían seguir alimentando la que P. Henríquez Ureña llamó, hace tiempo, «utopía americana».

\section{ALEJANDRO LOSADA: BIBLIOGRAFIA TENTATIVA *}

1966 Problemática del lenguaje (con J. L. Lazzarino y R. Palma de Carpinetti), Buenos Aires, Guadalupe.

1967 Martín Fierro. Gaucho, héroe, mito, Buenos Aires, Plus Ultra.

1968 Prólogo y Notas en Alfredo Ebelot, Recuerdos y relatos de la guerra de fronteras, Buenos Aires, Plus Ultra.

1969 Prólogo y Notas en José Hernández, Martín Fierro, Barcelona, Nauta.

\footnotetext{
* Las divergencias (poco importantes) que se encuentran en las bibliografías parciales consultadas no permiten garantizar, en todos los casos, la exactitud de la información proporcionada.
} 
1970 Andá cantále a Gardel. Novela, Buenos Aires, Aquarius.

1974 «La obra de J. M. Arguedas y la sociedad andina. Interpretación de su praxis literaria como praxis social», Eco (Bogotá), tomo 27, núm. 6-162, pp. 592-620.

1975a «Los sistemas literarios como instituciones sociales en América Latina», Revista de crítica literaria latinoamericana (Lima), núm. 1, pp. 39-61.

1975 b «Discursos críticos y proyectos sociales en América Hispánica», Acta litteraria (Budapest), núm. XVII, pp. 275-280.

1975c «Ciro Alegría como fundador de la realidad hispanoamericana», Acta litteraria (Budapest), núm. XVII, pp. 71-92.

1976a Creación y praxis. La producción literaria como praxis social en Hispanoamérica y el Perú, Lima, UNMSM.

1976b «structura social y producción cultural en América Latina. Las literaturas dependientes (1780-1920)», Actas del Simposio internacional de estudios hispánicos, Budapest, Hungarian Academy of Sciences, pp. 93-109.

1977 «Los modos de producción cultural de los estratos medios urbanos en América Latina: las culturas dependientes (1780-1920) y las culturas autónomas (1880-1970)», Revista de crítica literaria latinoamericana (Lima), núm. 6, pp. 7-36.

1978 «Bases para una estrategia de investigación del cambio cultural en América Latinas, Eco (Bogotá), tomo 32, núm. 196, pp. 337-374.

1979a "Rasgos específicos del realismo social en América Hispánica», Revista Iberoamericana (Pittsburgh), núms. 108-109, pp. 413-442.

1979b «El desarrollo de las culturas autónomas en América Latina. Ensayo de comprensión de los horizontes culturales de los intelectuales de América Latina como praxis de distintos grupos sociales», Bildung und Ausbildung in der Romania (ed. R. Kloepfer), Munich, Fink, tomo III, pp. 318-336.

1980 a «reación y praxis social en América Latina. La nueva narrativa como práctica de la marginalidad»; Iberoromania (Tübingen), Neu Folge, núm. 11, pp. 113-132.

1980 b «Cultura nacional o literatura revolucionaria? La producción cultural de los intelectuales autónomos en las sociedades periféricas», Nova Americana (Torino), pp. 287-330.

1980 c «El surgimiento del realismo social en la literatura de América Latina», Ideologies and Literature (Minnesota), núm. 9, pp. 20-55.

1981a La literatura en la sociedad de América Latina: I. Los modos de producción entre 1750 y 1980. Estrategias de investigación; Universidad de Odense, 2. ${ }^{\mathrm{a}}$ ed. corr. y aum.

1981b «Bases para un proyecto de una historia social de la literatura en América Latina», Revista Iberoamericana (Pittsburgh), núms. 114-115, pp. 167-188.

1891c César Vallejo. Actas del Coloquio Internacional en la Universidad Libre de Berlín (con G. Beutler), Tübingen, Niemeyer.

1982 «Funciones de los sistemas literarios marginales en los espacios metropolitanos. El caso Río de la Plata (1920-1980)», Perspectivas de comprensión y de explicación de la narrativa latinoamericana (ed. J. M. López de Abiada/ J. Penate Rivero), Bellinzona, Casagrande, pp. 113-132.

1983a La literatura en la sociedad de América Latina. Perí y el Río de la Plata, 1837-1880, Frankfurt/M., Vervuert.

1983b «Articulación, periodización y diferenciación de los procesos literarios en América Latinas, Revista de crítica literaria latinoamericana (Lima), núm. 17, pp. 7-37. 
1983c «La literatura marginal en el Río de la Plata, 1900-1960. Informe de investigación», Argentinien-Uruguay (ed. T. Heydenreich/J. Schneider), Munich, Fink, Lateinamerika-Studien 12, pp. 271-281.

1983d «La literatura latinoamericana en las metrópolis complejas. El caso CaribeAmérica Central entre 1920-1980», La literatura latinoamericana en el Caribe (ed. A. Losada), Berlín, Lateinamerika-Institut, pp. 266-351.

1983e «Sociedad y literatura en América Latina», número monográfico de la Revista de crítica literaria latinoamericana (Lima), núm. 17, coord. por A. Losada.

1984a «La literatura marginal en el Río de la Plata, 1900-1960», De los romancesvillancico a la poesía de Claudio Rodríguez (homenaje a G. Siebenmann), Madrid, J. Esteban, pp. 287-338.

1984b «La internacionalización de la literatura latinoamericana», Caravelle (Toulouse), núm. 42, pp. 15-40.

1984c Literatura y sociedad en América Latina: II. Modelos teóricos; Universidad de Aarhus (Dinamarca).

$1985 a$ «La historia social de la literatura latinoamericana», Actas AELSAL, Giessen, pp. 59-74.

1985b «¿Cómo puede un europeo estudiar la literatura latinoamericana?», Caravelle (Toulouse), p. 45.

1985c «La contribución de Angel Rama a la historia social de la literatura latinoamericana», Casa de las Américas (La Habana), núm. 150, pp. 44-57.

$1985 \mathrm{~d}$ Conferencia para el Congreso Dario-Martí, Managua, 1975 (mimeógrafo).

1985e Hacia una historia social de la literatura latinoamericana (coord. A. Losada/ Th. Bremer), Giessen (Actas AELSAL, 1983-1984).

1986 «Hacia una historia social de la literatura latinoamericana», número monográfico de la Revista de crítica literaria latinoamericana (Lima), núm. 23, coord. por A. Losada y Th. Bremer (no idéntico con 1985e). 